\title{
Terapia antirretroviral: investigação de implementação nos cuidados de saúde primários, Nampula, Moçambique
}

Paulo Das Neves Pires, ${ }^{1}$ Abdoulaye Marega, ${ }^{2}$ José Miguel Creagh ${ }^{2}$

\section{RESUMO}

Objetivo: Avaliar a situação clínica dos pacientes em terapia antirretroviral, as taxas de adesão e descontinuação e seus fatores determinantes e intervir para melhorar o programa de luta contra o vírus da imunodeficiência humana em Nampula.

Tipo de estudo: Investigação de implementação, descritiva mista.

Local: Província de Nampula, Moçambique, envolvendo 10 centros de saúde.

População: Pacientes em terapia antirretroviral e que descontinuaram, profissionais dos cuidados de saúde primários e praticantes tradicionais de saúde.

Métodos: Investigação de implementação, aplicando inquérito a pacientes e praticantes tradicionais de saúde, entrevista semiestruturada com profissionais de saúde e praticantes tradicionais e consulta documental. Avaliada a linha de base nos centros de saúde alvo e controlo foi realizada uma ação de educação para a saúde com os pacientes e com os praticantes tradicionais e de formação médica com os profissionais de saúde nos distritos alvo. Depois foram analisados os indicadores clínicos e do programa em todos os centros de saúde.

Resultados: A insegurança alimentar, discriminação e dificuldade de acesso aos serviços de saúde contribuem para uma taxa de descontinuação da terapia, atingindo $50 \%$ dos pacientes. A adesão terapêutica verifica-se em $69 \%$. A ação foi realizada em cinco distritos, com 63 pacientes, 59 praticantes tradicionais e 96 profissionais de saúde. Nos distritos de ação verificou-se um aumento do número de Grupos de Apoio à Adesão Comunitários e de referências aos cuidados de saúde primários, bem como uma melhoria relativa do estado clínico dos pacientes e da taxa de descontinuação da terapia, sem diferença estatisticamente significativa.

Conclusões: Existem diversas causas de descontinuação da terapia antirretroviral e de baixa adesão. A descontinuação da TARV é um problema grave em Nampula e resulta de fatores individuais, sociais e do sistema de saúde. Intervenções interdisciplinares de baixo custo na área da educação em saúde, associadas a extensão rural, podem inverter esta situação.

Palavras-chave: Antirretroviral; Cuidados de saúde primários; Descontinuação; Investigação de implementação; Moçambique; Vírus da imunodeficiência humana adquirida.

\section{INTRODUÇÃO}

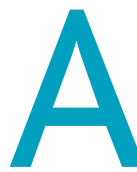
infeção pelo vírus da imunodeficiência humana (VIH) é um problema de saúde global: em 2016, 36,7 milhões de pessoas eram portadoras do VIH e a epidemia provocou 1,1 milhões de óbitos. África é a região mais afetada, com 25,6 milhões de portadores do VIH e 2/3 das novas infeções. ${ }^{1}$

Em 2015, Moçambique apresentava uma das maiores taxas de prevalência da infeção a nível mundial (13,2\%),

1. Secretário da Comissão Científica. Faculdade de Ciências de Saúde, Universidade Lurio.

2. Universidade Lúrio. sofrendo um impacto negativo da doença com 1,8 milhões de adultos infetados, ${ }^{2}$ dos quais 990.085 estavam em terapia antirretroviral (TARV). Este aumento significativo comparativamente aos 3.500 pacientes em TARV em 2003, resultante do esforço do Ministério da Saúde (MISAU) para garantir a TARV em todo o país ( $1 \%$ das unidades de saúde dispensavam TARV em 2003, 65\% em 2015), teve implicações sobre a carga das atividades nos cuidados de saúde primários (CSP) que carecem de profissionais de saúde (PS).

Em 2015, a província de Nampula apresentava uma prevalência deVIH (5,7\%) inferior à média nacional, com 
79.544 pessoas infetadas ( $69 \%$ em TARV) e a pior taxa de retenção em tratamento: $72 \%$ a 12 meses, $35 \%$ a 24 meses e $29 \%$ a 36 meses.

A TARV dispensada nos CSP em Moçambique transformou a síndroma de imunodeficiência humana adquirida (SIDA) em doença crónica, mediante uma boa adesão terapêutica (ingestão de comprimidos prescritos superior a $95 \%$ nos últimos três meses), necessária para a supressão viral; esta adesão varia no tempo e é influenciada por fatores diversos e complexos, um dos quais é fundamental, o atendimento no centro de saúde (CS), ${ }^{3}$ para garantir o acesso a medicamentos e serviços seguros, eficazes e de boa qualidade.

A transferência da prestação de cuidados aos pacientes portadores de VIH dos hospitais de dia para os CSP, em 2009, foi acompanhada por um elevado número de pacientes descontinuando a TARV, um desafio à saúde pública devido ao risco de resistência aos antirretrovirais (ARV).

Desde o início da campanha contra a SIDA, a Organização Mundial da Saúde recomenda como estratégia estabelecer uma relação com os praticantes tradicionais de saúde (PTS), sobretudo nos países de escassos recursos. Em África, estes «curandeiros» estão em todas as comunidades. Alguns referem os casos mais complicados para o Serviço Nacional de Saúde (SNS) Moçambicano, outros apoiam os PS na educação para a saúde dos pacientes que acedem aos CSP. O SNS abrange cerca de $40 \%$ da população do país, mas $100 \%$ desta frequenta os PTS. ${ }^{4}$ Nos locais onde ambas estão disponíveis, as pessoas utilizam a medicina tradicional e a moderna. ${ }^{5} \mathrm{Um}$ estudo Sul-africano mostra que os PTS são um recurso útil, mas subaproveitado, que pode apoiar o sistema biomédico ao colaborar na prevenção, no tratamento e referenciação, atenuando o impacto do VIH. ${ }^{6} \mathrm{Em}$ comunidades rurais, com baixa acessibilidade aos CSP, os PTS são fundamentais para aconselhar os portadores deVIH: eles educam a população com testemunhos pessoais, histórias e provérbios. Muitas pessoas referem que só acreditam na informação sobre o VIH quando esta é transmitida por estes praticantes. No entanto, até agora as ações que consideram e incluem esses atores são quase inexistentes. ${ }^{7}$

A compreensão dos sistemas que funcionam nas determinantes locais de um contexto específico, para uma população alvo, é essencial para garantir a expansão da $\mathrm{TARV}^{8}$ Algumas práticas socioculturais tradicionais transportam aspetos negativos (como a escarificação ritual e certos hábitos sexuais), contribuindo para a elevada in- cidência do VIH. Estas situações devem ser identificadas e revertidas em práticas positivas, para a prevenção da transmissão do vírus e da descontinuação da terapêutica, conforme proposto no Plano de Aceleração da Prevenção, Diagnóstico e Tratamento de VIH/SIDA. ${ }^{9}$

Até agora não existia na província de Nampula um conhecimento sistematizado sobre os determinantes de adesão terapêutica. ${ }^{10}$ Assim, esta investigação de implementação tinha como objetivo principal melhorar o programa TARV ${ }^{11-14}$ nesta província. Os objetivos secundários eram: a) avaliar a situação clínica dos pacientes em tratamento antirretroviral; b) avaliar as taxas de adesão e descontinuação e seus fatores determinantes; c) intervir para diminuir a descontinuação do TARV e melhorar a situação clínica dos pacientes em Nampula.

O estudo decorreu em três etapas:

1) Avaliar a adesão e a descontinuação (interrupção da toma de ARV por três meses ou mais) da TARV e os fatores determinantes nos CSP; planear métodos de adaptação e transferência de conhecimento para melhorar os indicadores do programa e o estado clínico dos pacientes; ${ }^{15-17}$

2) Aplicar as ações nos grupos alvos (pacientes, PS e PTS); ${ }^{18}$

3) Avaliar o impacto da intervenção na taxa de descontinuação da TARV, no estado clínico dos pacientes (índice de massa corporal [IMC] e ocorrência de infeções oportunistas) e na comunidade (grupos de apoio e referência de pacientes).

\section{MÉTODOS}

\section{Tipo de estudo}

Investigação de implementação, descritiva com abordagem mista (quantitativa e qualitativa) e ação de educação para a saúde. A componente quantitativa tem um desenho de estudo do tipo antes e depois com um grupo controlo, usando uma coorte de pacientes em TARV. Depois do estudo de linha de base, uma das coortes foi sujeita à ação (em cinco CS), a segunda (em outros cinco CS) constituiu o grupo controlo. O estudo foi realizado na província de Nampula, entre fevereiro de 2014 e setembro de 2015.

\section{Universo}

Distritos alvo: 8.251 pacientes inscritos em TARV nos CSP, em cinco CS das sedes distritais; respetivos PS responsáveis pelos serviços TARV (20) e PTS da área envol- 
vente. Distritos controlo: 6.904 pacientes inscritos em TARV nos CSP em cinco CS.

\section{Amostra e amostragem}

O estudo de linha de base (avaliação do estado clínico dos pacientes, adesão terapêutica e fatores determinantes) utilizou um número de pacientes a inquirir em cada distrito alvo (em tratamento e que descontinuaram), calculado utilizando a fórmula $n=\varepsilon^{2 *} p^{*}\left(1-\mathrm{q} / \mathrm{i}^{2}\right)$ em que ( $n=$ número de sujeitos da amostra, $\varepsilon=1,96, p=$ última prevalência da taxa de abandono da TARV no CS seja $12 \%, \mathrm{q}=1-p$, considerando um intervalo de confiança $=95 \%, \mathrm{i}=0,05$ de margem de erro). Adicionou-se uma margem de $10 \%$ para perdas ocasionais ou desistências, calculando 165 pacientes em TARV e 165 que descontinuaram. Os sujeitos foram escolhidos aleatoriamente, segundo uma data ao acaso de consulta TARV (pacientes em tratamento). O mesmo processo foi utilizado para calcular as amostras das coortes de ação e controlo. No estudo de linha de base foi utilizada a amostragem «bola de neve» para encontrar os pacientes que no mesmo dia tinham faltado à consulta ou ao levantamento de ARV na farmácia do CS há mais de três meses (descontinuaram o tratamento); seguiu-se busca ativa e assinatura de termo de consentimento informado (TCI).

Os PS responsáveis dos serviços TARV foram entrevistados individual e confidencialmente pelos investigadores, durante o estudo de linha de base e depois da ação. As entrevistas foram registadas em papel (não foram gravadas) e tratadas manualmente por tema (pacientes, TARV nos CSP, PTS).

Os PTS foram convocados pelo PS responsável no CS em articulação com o seu dirigente local e foram inquiridos individualmente durante o estudo de linha de base, com intérprete da língua local Macua, garantindo a confidencialidade. As respostas foram introduzidas e tratadas no programa SPSS, v. 21. Depois da ação foram entrevistados individualmente, com intérprete da língua local Macua, garantindo a confidencialidade e as entrevistas foram registadas em papel (não foram gravadas) e tratadas manualmente por tema (pacientes, TARV nos CSP, PTS).

\section{Critérios de inclusão}

1) Distritos: prevalência deVIH e taxa de abandono da TARV superior à média provincial, em pares classificados nas categorias geográficas utilizadas no Programa Na- cional de Combate ao VIH (urbano, rural, corredor, litoral); 2) pacientes: em TARV ou que descontinuaram a terapêutica, com processo clínico localizado, maior de idade, TCI assinado; 3) PS dos CSP: atividade principal no serviço TARV, disponibilidade e voluntariedade; 4) PTS: disponibilidade e voluntariedade documentada em TCI assinado.

\section{Variáveis}

Distritos: número de infetados porVIH e em TARV, número de pacientes que descontinuaram a TARV, incidência do VIH nas grávidas e nas consultas.

Pacientes: género, idade, em TARV ou descontinuou, escolaridade, posologia, ${ }^{19}$ depressão, consumo de psicotrópicos, ${ }^{20}$ apoio familiar, ${ }^{21}$ pertença a Grupo de Apoio à Adesão Comunitário (GAAC, associação de pacientes destinada a facilitar o acesso aos medicamentos e informação sobre o programa VIH), utilização dos PTS, conhecimento sobre os riscos da má adesão, levantamento de ARV na farmácia (últimos três meses), IMC, alimentação, falha da medicação, ${ }^{22-23}$ alegadas causas de descontinuação, ocorrência de diarreia ou vómitos e de tuberculose (TB) ou outra infeção oportunista.

Profissionais de saúde: género, idade, profissão, fatores percebidos de baixa adesão e descontinuação da TARV, dificuldades do programaVIH e sinergias possíveis com a comunidade para o melhorar.

Praticantes tradicionais de saúde: género, idade, tempo de prática, tratamento que usa, conceito e tratamento de TB e da infeção VIH, formação em VIH, conceito de adesão terapêutica, referência ao CS, conhecimento sobre alimentação.

\section{Implementação}

A investigação de implementação decorreu em três fases:

I) Estudo de base: seleção dos distritos de ação e controlo (fevereiro de 2014) por consulta documental e critérios geográficos (Quadro I): foram avaliados os indicadores do programa através dos registos da Direção Provincial de Saúde de Nampula (DPSN). Identificação dos grupos alvo (setembro de 2014): pacientes em TARV, pacientes que descontinuaram; consulta documental (estatística do CS e processos clínicos).

Recolha de dados no terreno (outubro de 2014): aplicação de inquérito aos pacientes pelos investigado- 


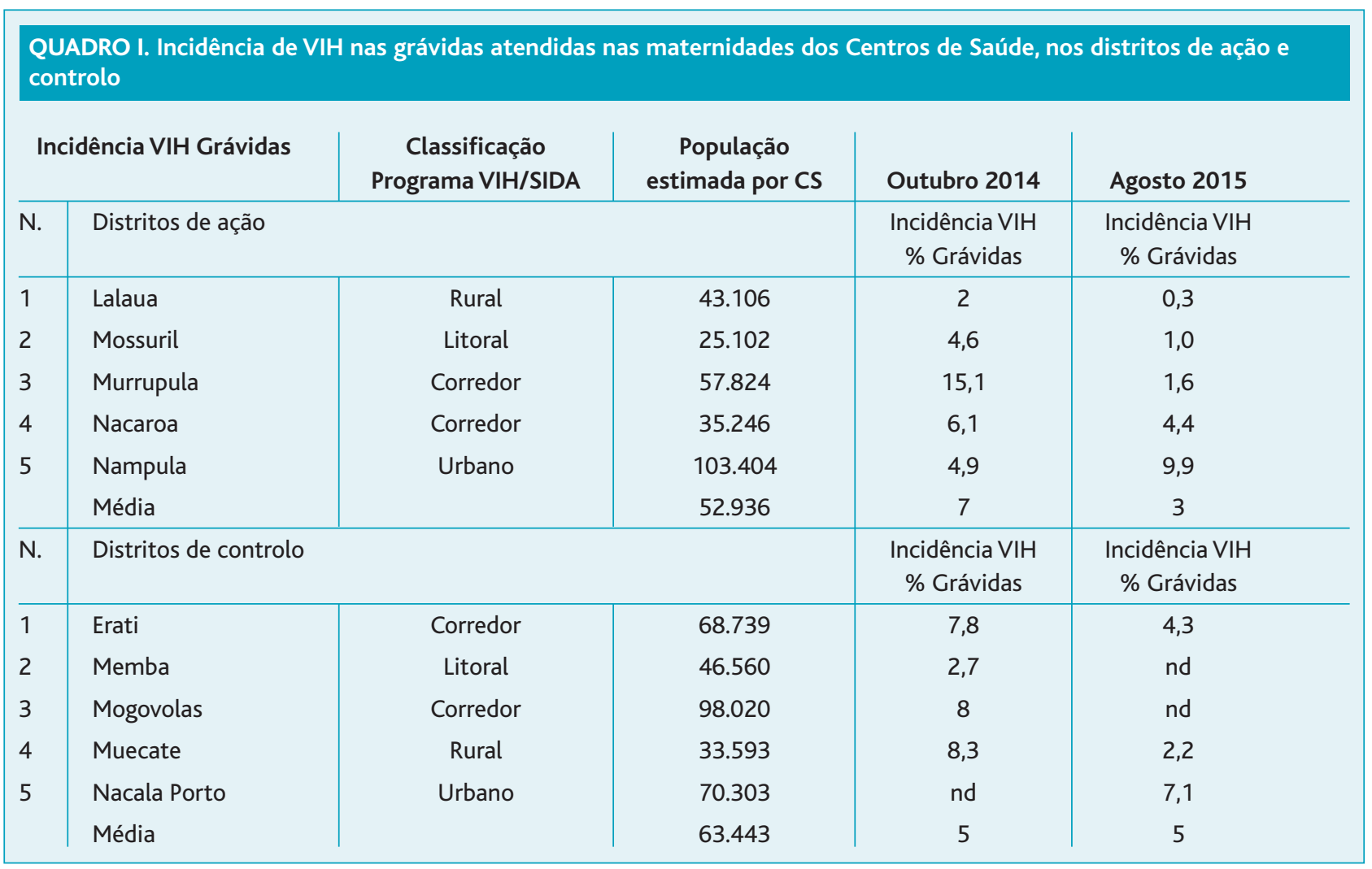

Legenda: $\mathrm{VIH}=$ vírus da imunodeficiência humana; SIDA = síndroma de imunodeficiência adquirida; $C S$ = centro de saúde; nd = resultados não disponíveis.

res, avaliação dos registos clínicos e de levantamento de ARV; foram selecionados sete inquiridores (ensino secundário completo, fluência na língua local, sem ligação com o SNS, desconhecidos dos inquiridos), treinados e assinado termo de compromisso ético para procederem às buscas ativas num raio pedestre do CS (5km), sendo pagos 100 meticais (dois euros) por cada inquérito realizado, acompanhado por um dos investigadores. Entrevista semiestruturada aos PS; não houve nenhuma recusa de participação e o registo foi reavaliado pelo entrevistado. Aplicação de inquérito pelos investigadores aos PTS. Tratamento da informação (dezembro de 2014): introdução de dados quantitativos no programa SPSS v. 21, tratamento e análise de dados respeitando um intervalo de confiança de $95 \%(p<0,05)$ e interpretação dos resultados. As entrevistas foram tratadas manualmente, agrupando a informação por tema (pacientes, serviços de saúde, PTS).

Plano de intervenção (março de 2015): elaboração de conteúdos (educação nutricional, doenças crónicas, adesão terapêutica, extensão agrária), de suportes didáticos e logísticos e plano de ação para os três grupos alvo.

II) Ação (abril de 2015): nos distritos de ação, os investigadores realizaram uma intervenção de formação médica com os PS e duas de educação para a saúde com os pacientes e familiares e com os PTS. Com os pacientes e com o apoio de um técnico de extensão agrária foram criados viveiros comunitários de hortícolas em locais perto do CS para aprendizagem e replicação. Foram reavaliados os indicadores do programa e dos pacientes em TARV em todos os 10 distritos e os dados introduzidos em ficheiro eletrónico Microsoft Office Excel e tratados em frequência, média e percentagem.

III) Avaliação (setembro de 2015): reavaliação dos indicadores do programa e dos pacientes nos mesmos distritos; os dados foram introduzidos em ficheiro eletrónico Microsoft Office Excel e tratados em fre- 
quência, média e percentagem e foi calculado o teste de qui-quadrado para tendências. Foram feitas entrevistas semiestruturadas aos PS e aos PTS; não houve nenhuma recusa de participação e as notas foram reavaliadas e confirmadas pelos entrevistados.

Viés

Foram considerados como fatores de viés a fiabilidade das respostas dos pacientes e PTS aos inquéritos e dos PS e PTS às entrevistas, optando-se pela triangulação de perguntas para evitar viés de aferição e de conclusão.

A adesão estimada pelo relato dos pacientes, útil em contextos de escassos recursos, está entre 6 a $10 \%$ acima da adesão estimada por doseamento farmacológico de ARV no sangue; ${ }^{19}$ assim, introduziu-se um fator de correção sobre a adesão encontrada de $-8 \%$.

\section{Considerações éticas}

O protocolo do estudo foi aprovado pelo Comité Institucional de Bioética para a Saúde da Universidade Lúrio e pela DPSN. O estudo respeitou todas as recomendações da Declaração de Helsínquia (2013).

Esta investigação foi registada pelo Ministério da Educação de Moçambique (AF-IV-PI-01 / FDI / $2^{\circ}$-21/2012). O relatório de atividades e os conteúdos das ações de formação médica e de educação para a saúde estão disponíveis mediante solicitação ao investigador principal.

\section{RESULTADOS}

\section{1) Fase 1 - Linha de base}

No início de 2014, os resumos estatísticos mensais do programa nos $10 \mathrm{CS}$ dos distritos em estudo mostravam uma incidência de VIH nas grávidas entre os 2 e os $10 \%$, taxas de cobertura da TARV de 24 a $61 \%$ e de descontinuação entre os 0 e os $21 \%$ (muito abaixo da realidade referida pelos profissionais de saúde e documentada nos processos clínicos avaliados).

\section{a) Pacientes}

Foram inquiridos 294 pacientes nos cinco distritos de ação, com idades entre os 18 e os 64 anos, $70 \%$ do género feminino. Destes, 208 (70,7\%) estavam em TARV e 86 tinham descontinuado o tratamento (dos quais $63 \%$ do género feminino). Seis dos 214 pacientes em TARV não aceitaram responder ao inquérito. Foram identificados 232 casos de descontinuação, dos quais $29 \%$ não tinham informação no processo para a busca ativa pelos PS ou voluntários de saúde comunitários; das 165 buscas ati- vas realizadas foram encontrados 99 pacientes, dos quais 13 não aceitaram responder ao inquérito; dos 86 pacientes que tinham descontinuado o tratamento, 60 (70\%) admitiram a referência ao CS para reiniciar a TARV.

As causas de descontinuação da TARV identificadas foram a insegurança alimentar (55 dos 86 pacientes que tinham descontinuado [64\%], 114 dos 205 pacientes em TARV [55,6\%], sem diferença estatisticamente significativa), o desemprego ( $97 \%$ dos pacientes que tinham descontinuado, $87 \%$ dos pacientes em TARV, $p=0,005$ ), o estigma e discriminação, o atendimento deficiente, a baixa escolaridade, o desconhecimento do risco de falha da medicação, a depressão, a falta de apoio familiar, o desconhecimento e a desconfiança dos GAAC.

No grupo em TARV, 144 (69\%) pacientes apresentaram adesão terapêutica ( $>95 \%$ das tomas nos últimos três meses).

\section{b) Profissionais de saúde}

Foram entrevistados 20 PS nos distritos de intervenção (3/4 do género masculino, média etária de 28 anos) sobre o funcionamento do programa TARV no seu CS: cinco médicos, 14 técnicos de saúde (seis enfermeiros, cinco técnicos de medicina curativa, dois técnicos de psiquiatria, um farmacêutico com formação de três anos nos Institutos de Ciências de Saúde) e um rececionista.

Problemas comuns identificados relativos à organização: processos clínicos mal preenchidos (4/5 CS); arquivo clínico desorganizado (5/5 CS); arquivo de levantamento de ARV na farmácia desorganizado (4/5 CS); tentativas infrutíferas de colaboração com os PTS (4/5 CS).

Perceções de justificação para a má adesão à TARV: grande número de pacientes descontinuando a TARV devido a desmotivação provocada por discriminação por familiares e amigos e à alimentação deficiente por falta de recursos económicos (5/5 CS).

\section{c) Praticantes tradicionais de saúde}

Foram inquiridos 79 PTS, incluindo parteiras tradicionais, $62 \%$ do género masculino, média etária de 38 anos, $65 \%$ com mais de 10 anos de prática.

A perda de peso é a principal suspeita de VIH mencionada por 26 (33\%), mas 20 (25\%) desconhecem os sinais; quase todos mencionaram não tratar estes pacientes e pensam que os ARV melhoram a sua qualidade de vida; 67 (85\%) tem uma ideia aproximada da boa adesão ("nunca falhar um dia nem atrasar mais de uma hora na toma dos comprimidos") e referem os casos complica- 
dos ao CS; 44 (56\%) tiveram formação sobre a doença.

\section{2) Fase 2 - Ação}

\section{a) Pacientes}

Foram realizadas cinco sessões de educação para a saúde com voluntários convocados pelo responsável do serviço TARV, com uma duração de cinco horas, sobre os temas: programa VIH, alimentação, doenças crónicas, adesão terapêutica e GAAC. Foi utilizada tradução simultânea, apoio audiovisual e distribuição de panfletos com imagens: 63 participantes (65\% do género feminino, idade média de 32 anos).

Foram implementados viveiros comunitários de hortícolas localizados perto do CS, com os mesmos pacientes e cinco técnicos de extensão agrícola, destinados a aumentar os recursos alimentares disponíveis para as famílias e melhorar o estado clínico dos pacientes.

Foram analisados os indicadores nos processos clínicos de 246 pacientes (146 nos distritos de ação, 100 nos distritos de controlo) e do programa no resumo estatístico mensal dos CS como linha de base antes da ação (Quadros I a IV).

\section{b) Profissionais de saúde}

Foram realizadas cinco sessões de formação (quatro horas) em língua portuguesa sobre o programa VIH, alimentação, adesão terapêutica, GAAC, com apoio audiovisual e distribuição de conteúdos em suporte papel: 96 participantes ( $5 \%$ do género feminino, idade média de 28 anos).

\section{c) Praticantes tradicionais de saúde}

Foram realizadas cinco sessões de formação (cinco horas) sobre o programaVIH, alimentação, doenças crónicas, adesão e GAAC. Foi usada tradução simultânea em língua local (Macua), apoio audiovisual e distribuição de panfletos com imagens: 59 participantes (47\% do género feminino, idade média de 39 anos).

\section{3) Fase 3 - Avaliação}

Foi realizada a recolha de indicadores do programa nos CS e nos processos clínicos de 348 pacientes (198 nos distritos de ação, 150 nos distritos de controlo). Os grupos de pacientes antes e depois da ação nos distritos de intervenção e nos distritos de controlo são comparáveis no que se refere ao género e à média etária (Quadro V). A análise final incidiu sobre os indicadores referentes aos meses de abril (antes da ação) e agosto (depois da ação) de 2015.

\section{a) Pacientes}

Nos distritos de ação, a incidência média da infeção em grávidas foi de $7 \%$ antes e de $3 \%$ após. Nos distritos de controlo manteve-se nos 5\% (Quadro I). Constatou-se uma melhoria em dois indicadores do estado clínico dos pacientes nos distritos de ação: aumento (1 ponto) na média do IMC (nos distritos de controlo não houve melhoria - Quadro II) e uma redução na ocorrência de infeções oportunistas de $1 \%$ (nos distritos de controlo registou-se um agravamento de $4 \%$ - Quadro III); estas diferenças, porém, não são estatisticamente significativas. A taxa de adesão terapêutica não sofreu alteração nos distritos de ação (de 69 para $68,2 \%$ ), tendo-se agravado nos distritos de controlo (de 62 para 55,3\%), sem diferença estatisticamente significativa. A taxa média de descontinuação aumentou $7 \%$ nos distritos de ação e $28 \%$ nos de controlo (Quadro IV), sem diferença estatisticamente significativa.

A implementação dos viveiros de hortícolas, para aprendizagem dos pacientes sobre os modos de sementeira e cultura, atingiu 55 sujeitos, abrangendo um total de $15.000 \mathrm{~m}^{2}$ e mostrando uma produção assinalável.

\section{b) Profissionais de saúde}

Foram entrevistados 10 PS voluntários, dois em cada CS. Estes referiram um retorno de pacientes que tinham descontinuado a TARV, sentem-se preparados para acompanhar a adesão terapêutica, mas não mobilizaram os PTS para as buscas ativas. O Quadro VI detalha a sua avaliação da ação, que conseguiu um aumento de $31 \%$ no número de GAAC funcionais (passou de 96 para 126 , contra 100 que se mantiveram nos distritos de controlo).

\section{c) Praticantes tradicionais de saúde}

Foram entrevistados 15 PTS voluntários, três em cada CS. Estes mobilizaram-se ativamente para a referência de doentes crónicos e grávidas ao CS (passaram de dois no último mês para 434). O Quadro VII resume a sua avaliação da ação.

\section{DISCUSSÃO}

\section{1) Pacientes}

A infeção pelo VIH mantém-se um desafio à saúde pública em Moçambique, mais grave nos centros urbanos e nas localidades «corredores» (situadas em vias principais de trânsito rodoviário). Os pacientes em TARV, sobretudo em meio rural, enfrentam muitas dificuldades. As causas identificadas de descontinuação da TARV nos 


\begin{tabular}{|c|c|c|c|c|c|}
\hline Distritos de ação & \multicolumn{2}{|c|}{ Antes (04/2015) } & \multicolumn{2}{|c|}{ Depois (08/2015) } & \multirow[b]{2}{*}{ Variação } \\
\hline $\begin{array}{l}\text { IMC médio dos } \\
\text { pacientes em TARV }\end{array}$ & $\begin{array}{l}\mathrm{N}^{\circ} \text { pacientes em } \\
\text { TARV avaliados IMC }\end{array}$ & IMC médio & $\begin{array}{l}\mathrm{N}^{\circ} \text { pacientes em } \\
\text { TARV avaliados IMC }\end{array}$ & IMC médio & \\
\hline Lalaua & 20 & 21 & 33 & 21 & 0 \\
\hline Mossuril & 20 & 21 & 38 & 22 & 1 \\
\hline Murrupula & 65 & 21 & 59 & 23 & 1 \\
\hline Nacaroa & 20 & 21 & 38 & 21 & -1 \\
\hline Nampula & 21 & 22 & 30 & 23 & 2 \\
\hline Total & 146 & 21 & 198 & 22 & 1 \\
\hline Distritos de controlo & \multicolumn{2}{|c|}{ Antes (04/2015) } & \multicolumn{2}{|c|}{ Depois (08/2015) } & \\
\hline $\begin{array}{l}\text { IMC médio dos } \\
\text { pacientes em TARV }\end{array}$ & $\begin{array}{l}N^{\circ} \text { pacientes em } \\
\text { TARV avaliados IMC }\end{array}$ & IMC médio & $\begin{array}{l}\mathrm{N}^{\circ} \text { pacientes em } \\
\text { TARV avaliados IMC }\end{array}$ & IMC médio & Variação \\
\hline Erati & 20 & 21 & 30 & 22 & 0 \\
\hline Memba & 20 & 22 & 30 & 23 & 1 \\
\hline Mogovolas & 20 & 19 & 30 & 21 & 2 \\
\hline Muecate & 20 & 22 & 30 & 20 & -3 \\
\hline Nacala Porto & 20 & 22 & 30 & 21 & -1 \\
\hline Total & 100 & 21 & 150 & 21 & 0 \\
\hline
\end{tabular}

Legenda: IMC = índice de massa corporal; TARV = tratamento antirretroviral.

inquéritos estão confirmadas na literatura: insegurança alimentar, desemprego, estigma e discriminação, atendimento deficiente no CS, baixa escolaridade, desconhecimento do risco de falha da medicação, depressão, falta de apoio familiar, desconhecimento e desconfiança dos GAAC. ${ }^{24-29}$

A alta frequência de insegurança alimentar destes pacientes está de acordo com a taxa de malnutrição na província de Nampula. Esta situação agravou-se em 2014 e 2015 devido à situação económica precária do país.

A confirmação da adesão relatada pelo sujeito é feita por triangulação, verificada no paciente que não teve atraso superior a três dias no levantamento de medicamentos na farmácia nos últimos três meses. ${ }^{30-34} \mathrm{~A}$ taxa média de adesão não sofreu alteração significativa, mantendo-se próxima do valor encontrado inicialmente, ${ }^{35-36}$ tendo sido, no entanto, agravada nos distritos de controlo.

O nível insuficiente de escolaridade traduz-se em fraco domínio da língua portuguesa e compreensão deficiente das mensagens de educação para a saúde, provocando taxas de descontinuação elevadas. A educação dos pacientes e a formação dos profissionais de saúde podem ter contribuído para diminuir a taxa de descontinuação da TARV nos distritos de ação relativamente aos distritos de controlo. No entanto, a diferença dos números de pacientes avaliados (nos distritos de ação e de controlo) e o facto dos sujeitos avaliados antes e depois da ação não serem os mesmos podem ter reduzido o impacto da ação.

\section{2) Profissionais de saúde}

Introduziram melhorias nos serviços, nomeadamente promovendo a criação de GAAC e facilitando o acesso dos pacientes à $\mathrm{TARV}^{37-38} \mathrm{o}$ que pode ter contribuído para diminuir o risco de descontinuação. Continua a verificar-se uma articulação insuficiente com os PTS, que não foram mobilizados para as buscas ativas e os PS não fazem contra referência dos pacientes enviados por aqueles.

\section{3) Praticantes tradicionais de saúde}

Demonstraram a sua disponibilidade para cooperar com os CSP e a sua capacidade de referir doentes crónicos e grávidas ao CS. 
QUADRO III. Evolução da incidência de infeções oportunistas nos pacientes em TARV, nos distritos de ação e de controlo

\begin{tabular}{|c|c|c|c|c|c|c|c|}
\hline \multirow{2}{*}{$\begin{array}{l}\text { Distritos de ação } \\
N^{\circ} \text { episódios } \\
\text { IO/paciente } \\
\text { em TARV }\end{array}$} & \multicolumn{3}{|c|}{ Antes (04/2015) } & \multicolumn{3}{|c|}{ Depois (08/2015) } & \multirow[b]{2}{*}{$\begin{array}{c}\text { Variação } \\
\text { percentual }\end{array}$} \\
\hline & $\begin{array}{c}\mathrm{N}^{\circ} \text { pacientes } \\
\text { em TARV } \\
\text { avaliados }\end{array}$ & $\begin{array}{c}N^{\circ} \text { em } \\
\text { TARV } \\
\text { com IO }\end{array}$ & $\begin{array}{c}\text { \% Pacientes } \\
\text { em TARV } \\
\text { com IO }\end{array}$ & $\begin{array}{c}N^{\circ} \text { pacientes } \\
\text { em TARV } \\
\text { avaliados }\end{array}$ & $\begin{array}{l}\text { No em } \\
\text { TARV } \\
\text { com IO }\end{array}$ & $\begin{array}{c}\% \text { Pacientes } \\
\text { em TARV } \\
\text { com IO }\end{array}$ & \\
\hline Lalaua & 20 & 0 & 0,0 & 33 & 7 & 21,2 & 21 \\
\hline Mossuril & 20 & 5 & 25,0 & 38 & 1 & 2,6 & -22 \\
\hline Murrupula & 65 & 11 & 16,9 & 59 & 7 & 11,9 & -5 \\
\hline Nacaroa & 20 & 0 & 0,0 & 38 & 12 & 31,6 & 32 \\
\hline Nampula & 21 & 9 & 42,9 & 30 & 5 & 16,7 & -26 \\
\hline Total & 146 & 25 & 17,1 & 198 & 32 & 16,2 & -1 \\
\hline $\begin{array}{l}\text { Distritos de } \\
\text { controlo }\end{array}$ & \multicolumn{3}{|c|}{ Antes (04/2015) } & \multicolumn{3}{|c|}{ Depois (08/2015) } & \\
\hline $\begin{array}{l}\text { No episódios } \\
\text { IO/paciente } \\
\text { em TARV }\end{array}$ & $\begin{array}{c}\mathrm{N}^{\circ} \text { pacientes } \\
\text { em TARV } \\
\text { avaliados }\end{array}$ & $\begin{array}{c}\mathrm{N}^{\circ} \text { em } \\
\text { TARV } \\
\text { com IO }\end{array}$ & $\begin{array}{c}\text { \% Pacientes } \\
\text { em TARV } \\
\text { com IO }\end{array}$ & $\begin{array}{c}N^{\circ} \text { pacientes } \\
\text { em TARV } \\
\text { avaliados }\end{array}$ & $\begin{array}{c}\mathrm{N}^{\circ} \mathrm{em} \\
\text { TARV } \\
\text { com IO }\end{array}$ & $\begin{array}{c}\text { \% Pacientes } \\
\text { em TARV } \\
\text { com IO }\end{array}$ & $\begin{array}{c}\text { Variação } \\
\text { percentual }\end{array}$ \\
\hline Erati & 20 & 3 & 15,0 & 30 & 4 & 13,3 & $-1,7$ \\
\hline Memba & 20 & 1 & 5,0 & 30 & 8 & 26,7 & 21,7 \\
\hline Mogovolas & 20 & 3 & 15,0 & 30 & 9 & 30,0 & 15,0 \\
\hline Muecate & 20 & 9 & 45,0 & 30 & 6 & 20,0 & $-25,0$ \\
\hline Nacala Porto & 20 & 0 & 0,0 & 30 & 3 & 10,0 & 10,0 \\
\hline Total & 100 & 16 & 16,0 & 150 & 30 & 20,0 & 4,0 \\
\hline
\end{tabular}

Legenda: TARV = tratamento antirretroviral; IO = infeção oportunista.

\section{4) Limitações do estudo}

1. A informação oficial disponível sobre descontinuação da TARV é limitada e muitas vezes incorreta.

2. Os arquivos clínicos dos pacientes estão desorganizados e com falta de informação, tendo sido recomendado às Direções dos CS um registo exaustivo e a organização dos ficheiros com identificação dos pacientes que descontinuaram a TARV.

3. O número de pacientes inquiridos que descontinuaram a TARV foi inferior à amostra calculada, diminuindo a significância estatística das razões alegadas para descontinuação.

4. As coortes avaliadas antes e depois da ação são compostas de sujeitos diferentes, impedindo a estimativa precisa do impacto sobre a adesão à TARV.

5. A articulação interprofissional a nível dos CSP e interinstitucional nos distritos e com o nível provincial é deficiente, ${ }^{39}$ limitando o impacto da ação de extensão rural.

\section{5) Generalização}

As ações realizadas reduziram a probabilidade de descontinuação da TARV, podendo considerar-se como pontos positivos:

1) Impacto psicológico nos pacientes, que se sentiram reconhecidos, sem discriminação e sensibilizados para a importância da boa adesão, como meio de prevenção da infeção.

2) Impacto técnico nos PS, que reconheceram progressos na capacidade de acompanhar a adesão e prevenir a descontinuação - referiram ter reiniciado a TARV em muitos pacientes.

3) Melhoria da articulação dos PTS com os CSP recuperando muitos pacientes que tinham descontinuado a TARV.

4) Hortas comunitárias, educando e incentivando as famílias ao cultivo nas suas machambas e à introdução de novas culturas (e.g. alface, beringela, cenoura). 


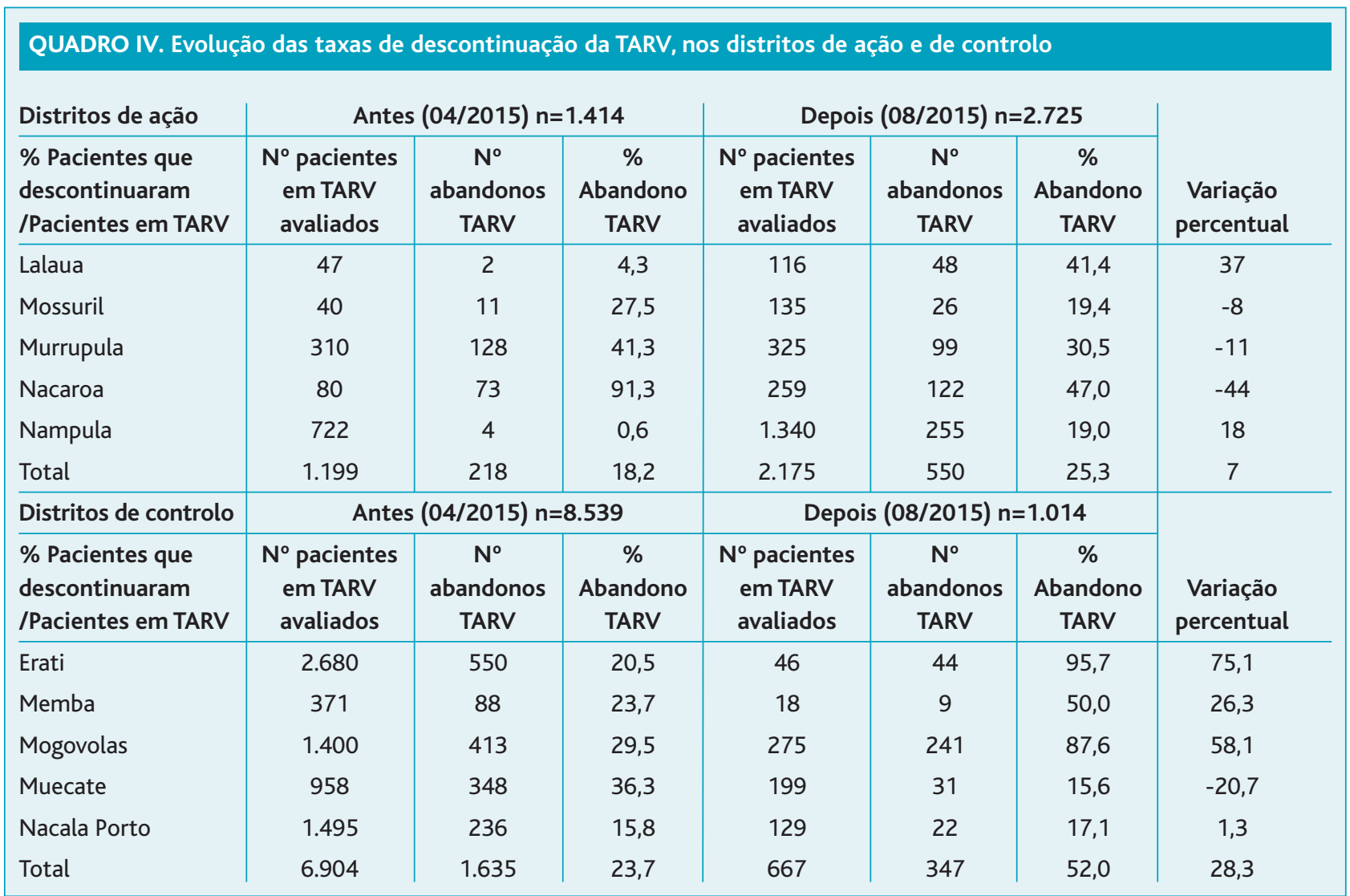

Legenda: TARV = tratamento antirretroviral; $n=$ número de pacientes avaliados.

\begin{tabular}{|c|c|c|c|c|}
\hline \multirow[b]{2}{*}{ Distritos } & \multicolumn{2}{|c|}{ Antes da ação: $n=9.953$} & \multicolumn{2}{|c|}{ Depois da ação: $n=3.739$} \\
\hline & $\begin{array}{c}\text { \% Sujeitos do género } \\
\text { feminino }\end{array}$ & $\begin{array}{c}\text { Média etária } \\
\text { (anos) }\end{array}$ & $\begin{array}{c}\% \text { Sujeitos do género } \\
\text { feminino }\end{array}$ & $\begin{array}{l}\text { Média etária } \\
\text { (anos) }\end{array}$ \\
\hline Controlo & 69,0 & 28,9 & 69,3 & 28,1 \\
\hline Ação & 72,6 & 30,6 & 71,7 & 28,4 \\
\hline
\end{tabular}

Legenda: $n=$ número de pacientes avaliados.

As características da população e dos serviços de saúde avaliados reproduzem a situação geral a nível provincial. As ações realizadas foram demonstradas pela literatura internacional como sendo eficazes e os resultados conseguidos melhoraram o programa contra o VIH nos CSP. No contexto Moçambicano seria necessário promover a formação dos PS e a educação e extensão rural com os pacientes e agentes locais, de acordo com os resultados deste estudo, para obter uma cobertura sanitária universal. ${ }^{40}$

\section{6) Conclusão}

A alta taxa de descontinuação da TARV em Nampula pode ser responsável pela manutenção da epidemia. Este fenómeno é preocupante e está subavaliado, mas pode ser revertido.

A educação dos pacientes e a extensão rural podem reduzir a taxa de descontinuação da TARV e ter um efeito benéfico sobre o estado clínico dos pacientes. No entanto, a sua participação nestas atividades é limitada por dificuldades de acesso aos CSP e estigmatização, necessitando de uma estratégia de comunicação eficaz. Os PTS 
QUADRO VI. Avaliação do estudo ação pelos profissionais de saúde (setembro de 2015)

\begin{tabular}{|c|c|}
\hline Questão & Comentários \\
\hline $\begin{array}{l}\text { Que mudanças } \\
\text { positivas foram } \\
\text { verificadas depois } \\
\text { da ação de educação } \\
\text { para a saúde? }\end{array}$ & $\begin{array}{l}\text { Pacientes: } \\
\text { Melhorou a adesão à testagem VIH, às sessões de educação para a saúde e à TARV em geral, sobretudo } \\
\text { nos serviços de saúde materna e TB. } \\
\text { Aumentou o recurso ao CS no caso de infeções oportunistas. } \\
\text { Grupos de pacientes iniciaram cultivo de legumes e estão a produzir. } \\
\text { Centro de saúde: } \\
\text { Distribui pacotes alimentares, trimestralmente, aos pacientes em TARV. } \\
\text { Todos os pacientes que vêm à consulta TARV passam obrigatoriamente no aconselhamento sobre adesão } \\
\text { terapêutica, que foi melhorado. } \\
\text { A disponibilização de ARV para seis meses na Farmácia melhorou a adesão e melhorou a articulação e } \\
\text { transferência de informação com o serviço TARV. } \\
\text { Foram organizados novos GAAC. } \\
\text { Os «faltosos» são detetados diariamente, contactados telefonicamente para busca ativa, resultando na } \\
\text { diminuição do número de «faltosos» e em muitos reinícios de TARV. } \\
\text { Pacientes em TARV tiveram formação em Educadores de Pares e estão a realizar busca ativa. } \\
\text { Lançaram busca ativa com PTS, PS e ativistas das organizações comunitárias de base. } \\
\text { Praticantes tradicionais de saúde: } \\
\text { Melhorou a referência de pacientes em geral aos CSP pelos PTS. }\end{array}$ \\
\hline $\begin{array}{l}\text { Que dificuldades } \\
\text { ainda persistem? }\end{array}$ & $\begin{array}{l}\text { Pacientes: } \\
\text { Falta de transporte para o CS dos pacientes residentes a grandes distâncias (mais de } 20 \mathrm{~km} \text { ). } \\
\text { Carência alimentar por falta de recursos financeiros e falsos conceitos sobre o tratamento prejudicam a } \\
\text { adesão. } \\
\text { Falta de apoio familiar impede os doentes incapacitados de receberem ARV. } \\
\text { Grávidas testadas recusam o diagnóstico e as que iniciam TARV durante a gravidez descontinuam a TARV } \\
\text { depois do parto. } \\
\text { A compreensão dos pacientes das mensagens educativas é reduzida, dificultando o combate ao estigma. } \\
\text { Adesão à TARV é oscilante e muitos pacientes descontinuam no início. } \\
\text { Pacientes são discriminados pelas famílias. } \\
\text { Conceito negativo sobre a doença, gera estigma e discriminação impedindo a formação de GAAC. } \\
\text { Pacientes reclamam dos efeitos secundários com a nova linha de TARV. } \\
\text { Os legumes do viveiro não foram disseminados pelos grupos de pacientes. } \\
\text { Falta de apoio das organizações comunitárias de base para os GAAC. } \\
\text { Centro de saúde: } \\
\text { Receção em más condições e rececionista sem formação adequada. } \\
\text { Não existe na maioria dos CS uma cesta básica de alimentos para motivar os pacientes a levantar os } \\
\text { ARV. } \\
\text { Atitude e comunicação deficiente em alguns PS estigmatiza o paciente. } \\
\text { Paciente demora um tempo exagerado no CS para levantar os ARV. } \\
\text { Registo da farmácia não permite um acompanhamento correto do paciente. } \\
\text { As buscas ativas são dificultadas por falta de informação sobre o paciente, falta de ativistas e de meios } \\
\text { de deslocação às comunidades. } \\
\text { Falta de manutenção, organização e apoio da administração local ao CS. } \\
\text { Praticantes tradicionais de saúde: } \\
\text { Necessitam de guias de referência para enviar os pacientes ao CS. }\end{array}$ \\
\hline
\end{tabular}

Legenda: $\mathrm{VIH}=$ vírus da imunodeficiência humana; TARV = tratamento antirretroviral; $\mathrm{TB}$ = tuberculose; $\mathrm{CS}$ = centro de saúde; GAAC = grupo de apoio à adesão comunitário; PTS = praticante tradicional de saúde; PS = profissional de saúde; $C S P$ = cuidados de saúde primários; $A R V=$ medicamentos antirretrovirais. 


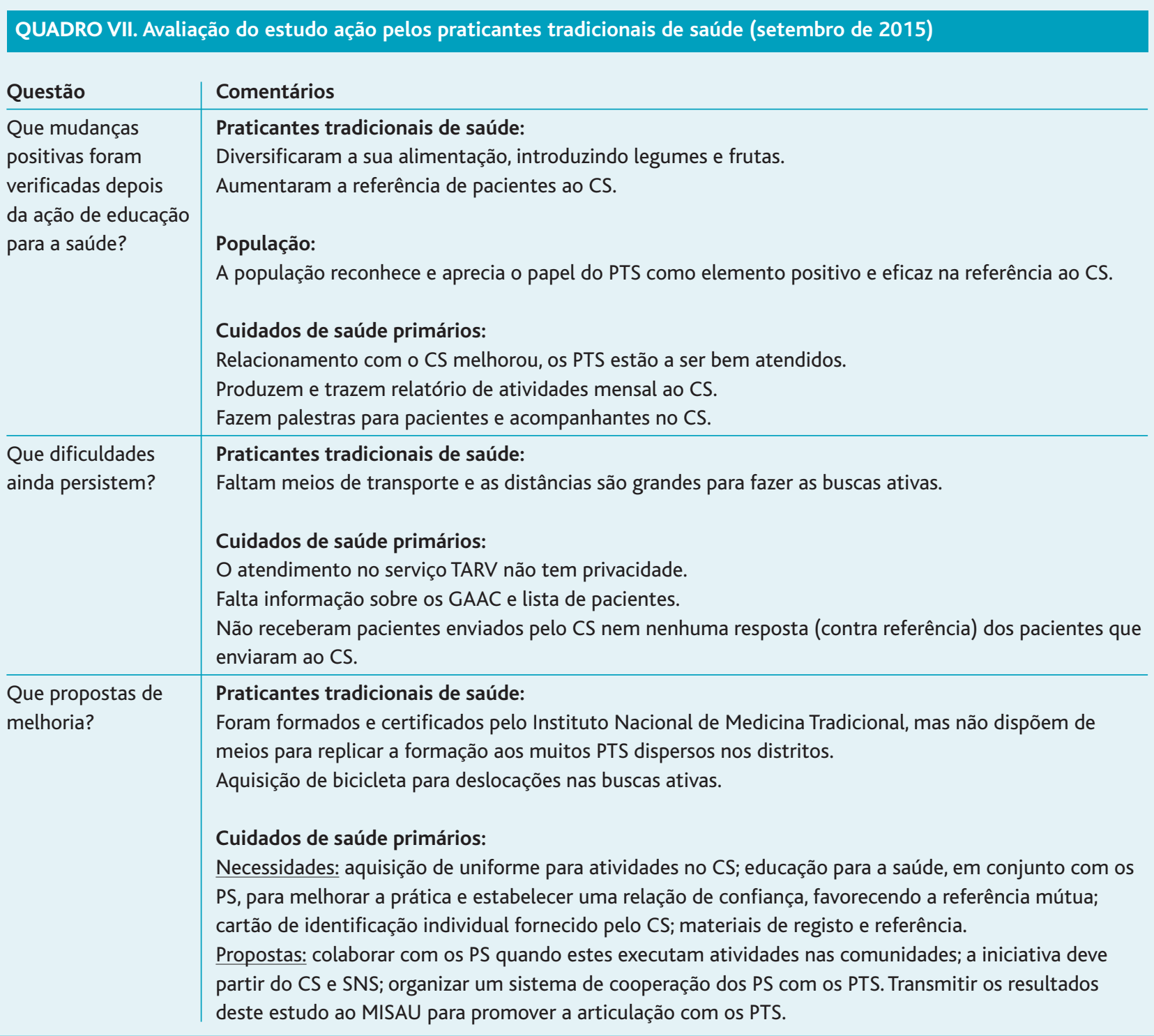

Legenda: $\mathrm{CS}$ = centro de saúde; PTS = praticante tradicional de saúde; TARV = tratamento antirretroviral; GAAC = grupo de apoio à adesão comunitário; PS = profissional de saúde; SNS = Serviço Nacional de Saúde; MISAU = Ministério da Saúde da República de Moçambique.

são parceiros dos CSP e representam um recurso subutilizado; reforçar o investimento nesta parceria poderá ter um impacto positivo no programa VIH. Os PS, após formação dirigida aos problemas identificados, melhoram a qualidade do atendimento aos pacientes.

Considerando a gravidade da infeção pelo VIH em Moçambique, seria necessário generalizar a formação conjunta dos PS e dos PTS, dos pacientes e familiares e promover a extensão rural em todos os CS com serviço TARV.

\section{REFERÊNCIAS BIBLIOGRÁFICAS}

1. Organización Mundial de la Salud.VIH-sida: datos y cifras [homepage]. Genebra: OMS; 2018 [updated 2018 Jul 19]. Available from: http://www. who.int/es/news-room/fact-sheets/detail/hiv-aids

2. Ministério da Saúde. Relatório anual 2016: relatório anual das atividades relacionadas ao HIV/SIDA. Maputo: MISAU; 2017.

3. Marega A, Pires P, Samuel J. Antiretroviral treatments abandon determinants in HIV positive patients, Chiúre, Mozambique, 2015. Int J Res. 2017;4(3):219-36.

4. Matsinhe C. Tabula rasa: dinâmica da resposta Moçambicana ao 
HIV/SIDA. Maputo: Texto Editores; 2006.

5. Lubinga SJ, Kintu A, Atuhaire J, Asiimwe S. Concomitant herbal medicine and Antiretroviral Therapy (ART) use among HIV patients in Western Uganda: a cross-sectional analysis of magnitude and patterns of use, associated factors and impact on ART adherence. AIDS Care. 2012;24 (11):1375-83.

6. George G, Chitindingu E, Gow J. Evaluating traditional healer's knowledge and practices related to HIV testing and treatment in South Africa. BMC Int Health Hum Rights. 2013;13:45.

7. Organisation Mondiale de la Santé. Rapport de la consultation sur le SIDA et la médecine traditionnelle: contribution possible des tradipraticiens (Francistown, Botswana, 23-27 juillet 1990). Genève: OMS; 1990.

8. Sandelowski M, Voils $\mathrm{Cl}$, Chang Y, Lee EJ. A systematic review comparing antiretroviral adherence descriptive and intervention studies conducted in the USA. AIDS Care. 2009;21(8):953-66.

9. Ministério da Saúde. Plano de aceleração da prevenção, diagnóstico e tratamento de HIV/SIDA (2013-2015). Maputo: MISAU; 2012.

10. Nachega JB, Mills EJ, Schechter M.Antiretroviral therapy adherence and retention in care in middle-income and low-income countries: current status of knowledge and research priorities. Curr Opin HIV AIDS. 2010;5 (1):70-7.

11. Pantoja T, Opiyo N, Lewin S, Paulsen E, Ciapponi A, Wiysonge CS, et al. Implementation strategies for health systems in low-income countries: an overview of systematic reviews. Cochrane Database Syst Rev. 2017;9: CD011086.

12. Marcellin F, Spire B, Carrieri MP, Roux P. Assessing adherence to antiretroviral therapy in randomized HIV clinical trials: a review of currently used methods. Expert Rev Anti Infect Ther. 2013;11(3):239-50.

13. Williams AB, Amico KR, Bova C, Womack JA.A proposal for quality standards for measuring medication adherence in research. AIDS Behav. 2013;17(1):284-97.

14. Peltzer K, Pengpid S. Socioeconomic factors in adherence to HIV therapy in low- and middle-income countries.J Health Popul Nutr.2013;31(2):150-70.

15. Tang AM, Quick T, Chung M, Wanke CA. Nutrition assessment, counseling, and support interventions to improve health-related outcomes in people living with HIV/AIDS: a systematic review of the literature. J Acquir Immune Defic Syndr. 2015;68 Suppl 3:S340-9.

16. van der Horst C, Hudson FP. Primary care for HIV-infected patients in resource-limited settings [homepage]. InPractice.com; 2018. Available from: https://www.inpractice.com/Textbooks/HIV/General_Approach_to_the_HIVInfected-Patient/ch9_Primary_Care_ResourceLimited/Chapter-Pages/Page-1

17. Côté JK, Godin G. Efficacy of interventions in improving adherence to antiretroviral therapy. Int J STD AIDS. 2005;16(5):335-43.

18. Amico KR, Orrell C. Antiretroviral therapy adherence support: recommendations and future directions. J Int Assoc Provid AIDS Care. 2013;12 (2):128-37.

19. Sullivan PS, Campsmith ML, Nakamura GV, Begley EB, Schulden J, Nakashima AK. Patient and regimen characteristics associated with selfreported nonadherence to antiretroviral therapy. PLoS One. 2007;2(6):e552.

20. Azar MM, Springer SA, Meyer JP, Altice FL. A systematic review of the impact of alcohol use disorders on HIV treatment outcomes, adherence to antiretroviral therapy and health care utilization. Drug Alcohol Depend. 2010;112(3):178-93.
21. Stubbs BA, Micek MA, Pfeiffer JT, Montoya P, Gloyd S. Treatment partners and adherence to HAART in Central Mozambique.AIDS Care. 2009; 21(11):1412-9.

22. Berg KM, Arnsten JH. Practical and conceptual challenges in measuring antiretroviral adherence. J Acquir Immune Defic Syndr. 2006;43 Suppl 1:S79-87.

23. Bulgiba, Mohammed UY, Chik Z, Lee C, Peramalah D. How well does self-reported adherence fare compared to therapeutic drug monitoring in HAART? Prev Med. 2013;57 Suppl:S34-6.

24. Pires PN, Marega A, Creagh JM. Adesao à terapia antirretroviral em pacientes infetados pelo $\mathrm{VIH}$ nos cuidados de saúde primários em Nampula, Mocambique [Adherence to antiretroviral treatment among HIV positive patients in primary health care in Nampula, Mozambique]. Rev Port Med Geral Fam. 2017;33(1):30-40. Portuguese

25. Pires $P$, Marega A, Creagh J. Food insecurity and ART non-adherence, Nampula, Mozambique. Int J Res. 2015;2(12):92-8.

26. Rachlis BS, Mills EJ, Cole DC. Livelihood security and adherence to antiretroviral therapy in low and middle-income settings: a systematic review. PLoS One. 2011;6(5):e18948.

27. Nischal KC, Khopkar U, Saple DG. Improving adherence to antiretroviral therapy. Indian J Dermatol Venereol Leprol. 2005;71(5):316-20.

28. Reisner SL, Mimiaga MJ, Skeer M, Perkovich B, Johnson CV, Safren SA. A review of HIV antiretroviral adherence and intervention studies among HIV-infected youth. Top HIV Med. 2009;17(1):14-25.

29. Bigler S, Nicca D, Spirig R. Welche Interventionen bewirken eine Verbesserung der Adhärenz von HIV-Patienten unter ART? [Interventions to enhance adherence of patients with HIV on ART: a literature review]. Pflege. 2007;20(5):268-77. German

30. Reynolds N. Optimizing adherence to antiretroviral therapy. InPractice.com [Internet]; 2015 [updated 2018 Mar 21]. Available from: https://www.inpractice.com/Textbooks/HIV/Antiretroviral_Therapy/ch 13_pt1_Adherence.aspx

31. Wasti SP, van Teijlingen E, Simkhada P, Randall J, Baxter S, Kirkpatrick $P$, et al. Factors influencing adherence to antiretroviral treatment in Asian developing countries: a systematic review. Trop Med Int Health. 2012;17(1):71-81.

32. Leeman J, Chang YK, Lee EJ, Voils Cl, Crandell J, Sandelowski M. Implementation of antiretroviral therapy adherence interventions: a realist synthesis of evidence. J Adv Nurs. 2010;66(9):1915-30.

33. Machtinger EL, Bangsberg DR. Seven steps to better adherence: a practical approach to promoting adherence to antiretroviral therapy. AIDS Read. 2007;17(1):43-51.

34. Berg KM, Arnsten JH. Practical and conceptual challenges in measuring antiretroviral adherence. J Acquir Immune Defic Syndr. 2006;43 Suppl 1:S79-87.

35. Simoni JM, Amico KR, Smith L, Nelson K. Antiretroviral adherence interventions: translating research findings to the real-world clinic. Curr HIV/AIDS Rep. 2010;7(1):44-51.

36. Mills EJ, Nachega JB, Buchan I, Orbinski J, Attaran A, Singh S, et al. Adherence to antiretroviral therapy in sub-Saharan Africa and North America: a meta-analysis. JAMA. 2006;296(6):679-90.

37. Soeters H, Hatane L, Gittings L. Community caregiver support groups to improve ART adherence amongst adolescents living with HIV [Internet]. Cape Town: Team PATA Promising Practices; 2016. Available 
from: http://teampata.org/wp-content/uploads/2017/06/MmataTswana_V1.pdf

38. Joint United Nations Programme on HIV/AIDS (UNAIDS). Community-based antiretroviral therapy delivery: experiences of Médecins sans Frontières [Internet]. New York: UNAIDS; 2015. Available from: http://www.unaids.org/sites/default/files/media_asset/20150420_MSF _UNAIDS_JC2707.pdf

39. Kadri MR, Schweickardt J. A emergência da Aids no Amazonas [The emergence of Aids in Amazonas]. Hist Cienc Saúde - Manguinhos. 2016;23(2):301-19. Portuguese

40. Organisation Mondiale de la Santé. Rapport sur la santé dans le monde 2013: la recherche pour la couverture sanitaire universelle. Genève: OMS; 2013. ISBN 9789240691216

\section{FINANCIAMENTO}

O estudo teve um custo total de 683.042 meticais (9.758 euros), finan- ciado em $79 \%$ pelo Fundo de Desenvolvimento Institucional do Ministério da Educação de Moçambique e em 21\% pela Universidade Lúrio, sem qualquer outra intervenção dos financiadores.

\section{CONFLITOS DE INTERESSE}

Os autores declaram não possuir quaisquer conflitos de interesse para a realização deste trabalho ou redação do artigo.

\author{
ENDEREÇO PARA CORRESPONDÊNCIA \\ Paulo Das Neves Pires \\ E-mail: druidatom@gmail.com \\ orcid.org/0000-0002-2586-9955
}

Recebido em 19-02-2018

Aceite para publicação em 28-07-2018

\section{ABSTRACT}

\section{ANTIRETROVIRALTREATMENT: PRIMARY HEALTH CARE IMPLEMENTATION RESEARCH, NAMPULA, MOZAMBIQUE}

Aim: To evaluate the clinical status of HIV-infected patients under antiretroviral therapy, rates of adherence and discontinuation of medication, and their determinants, aiming to improve the program to fight human immunodeficiency virus in Nampula.

Study: Implementation research, descriptive with mixed methods.

Place: Nampula Province, Mozambique, involving 10 health centres.

Population: Patients on antiretroviral therapy and those who discontinued therapy, primary health care professionals, and traditional healers

Methods: Implementation research, applying surveys to patients and traditional healers, semi-structured interviews to health professionals and traditional healers, and documental review. Baseline evaluation was performed in both target and control primary health care centres, followed by health education sessions for patients and traditional healers, and training of health care professionals in target health centres. Finally, we compared clinical and program indicators in all health centres.

Results: Food insecurity, discrimination and low accessibility to health care contribute to a discontinuation of antiretroviral therapy in up to $50 \%$ of patients. Therapeutic adherence is observed in $69 \%$ of the patients. The intervention was carried out in five districts, comprising 63 patients, 59 traditional healers and 96 health professionals. The intervention districts showed more community adherence support groups, higher number of patients referred to primary health care services, and achieved better patient clinical indicators and a lower treatment discontinuation rate, although not statistically significant.

Conclusion: There are several reasons for antiretroviral therapy discontinuation and low adherence rates. Discontinuation of antiretroviral therapy is a severe problem in Nampula due to individual, social and health system factors. Interdisciplinary and low-cost interventions such as health education with extension to rural areas may change this scenario.

Keywords: Discontinuation; Antiretroviral; Primary health care; Implementation research; Mozambique; Human immunodeficiency virus. 